\title{
Housekeeping genes involved in non-malignant breast phenotypes are widely expressed in multiple cancers and provide novel biomarkers of tumor classification
}

\author{
L. Delmonico ${ }^{1 * \oplus \otimes}$, J.C. Obenauer ${ }^{2 * \oplus}$, T.P. Stockfisch ${ }^{2 \oplus}$, and M.V. Fournier ${ }^{3 \oplus}$ \\ ${ }^{1}$ Instituto de Biofísica Carlos Chagas Filho, Universidade Federal do Rio de Janeiro, Rio de Janeiro, RJ, Brasil \\ ${ }^{2}$ Rancho BioSciences, San Diego, CA, USA \\ ${ }^{3}$ Bioarray Genetics Inc., Farmington, CT, USA
}

\begin{abstract}
Clinically relevant biomarkers are useful to determine cancer patients' prognosis and treatments. To discover new putative biomarkers, we performed in silico analysis of a 325-gene panel previously associated with breast epithelial cell biology and clinical outcomes. Sixteen public datasets of microarray samples representing 8 cancer types and a total of 3,663 patients' samples were used for the analyses. Feature selection was used to identify the best subsets of the 325 genes for each classification, and linear discriminant analysis was used to quantify the accuracy of the classifications. A subset of 102 of the 325 genes were found to be housekeeping (HK) genes, and the classifications were repeated using only the 102 HK subset. The 325-gene panel and $102 \mathrm{HK}$ subset were able to distinguish colon, gastric, lung, ovarian, pancreatic, and prostate tumors and leukemia from normal adjacent tissue, and classify disease subtypes of breast and lung cancers and leukemia with $70 \%$ or higher accuracy. HK genes have been overlooked as potential biomarkers due to their relative stability. This study describes a set of HK genes as putative biomarkers applicable to multiple cancer types worth following in subsequent validation studies.
\end{abstract}

Key words: Cancer biomarkers; Housekeeping genes; Cancer gene profiling; RNA biomarkers; Oncology biomarkers

\section{Introduction}

Oncogenes and other genes involved in carcinogenesis are well-studied candidates for biomarkers, but some researchers have also looked at models of normal growth, differentiation, or development to identify cancer-relevant genes with some success (1-4). In this context, housekeeping genes (HK) have become a target of research for their relative stability regardless of cell development stages, specific tissue, or external conditions $(5,6)$.

$\mathrm{HK}$ are essential genes for basal cell maintenance, regardless of tissue of origin (5). These genes have different evolutionary profiles than others, which contribute to genomic stability. In a previous study by our group, a set of 325 genes was identified as being involved in the formation of organized ductal units in 3-dimensional human mammary epithelial cell culture in laminin-rich extracellular matrix (7). This process includes the transition of cells from a disorganized proliferating state to an organized growth-arrested and polarized state, and these same genes were used successfully to classify breast cancer patients into good and poor prognosis groups $(7,8)$. It has recently been shown that a subset of the 325 gene panel was able to stratify triple negative breast cancer patients into responders to neoadjuvant chemotherapy (NAC), minimal residual disease (RD) after NAC, and even worse-surviving RD cases (9). Moreover, the reproducibility of the 325 RNA biomarkers was validated by comparing two gene expression platforms (Affymetrix and NanoString) (10).

Interestingly, when extending the evaluation of this signature to determine the best method for batch correction of microarray data, it was discovered that a set of 102 genes of the 325 genes are classified as HK (11). A highly cited list of HK genes was defined by the Levanon lab in 2003 (5) and was updated in 2013 (6). In the latter study, the authors identified 3,804 human HK genes, using the criteria that they showed less than four-fold variation in

Correspondence: L. Delmonico: <lucasdelmonico@gmail.com>

${ }^{*}$ These authors contributed equally to this study.

Received June 12, 2020 | Accepted February 12, 2021 
expression across 16 normal human tissues. Then, in this study, the 325 genes were tested for their ability to be used as RNA expression biomarkers in other cancer types besides breast, using either of two criteria: 1) having different expression levels in tumors than in healthy tissue; or 2) having different expression in subtypes of the same cancer. After the initial tests of biomarker capability using the 325 genes, the tests were repeated on the HK subset (102 genes) to determine whether HK genes by themselves can classify tumors.

\section{Material and Methods}

\section{List of the 325 genes}

Samples information from 16 cancer data sets were extracted from the SOFT files for GEO studies (such as GSE26712_family.soft). Sixteen cancer data sets obtained from GEO and ArrayExpress were used to evaluate the expression of the 325 genes (Table 1). For the one ArrayExpress study (E-TABM-157), sample information was extracted from its SDRF file and its associated publication (12). The gene symbols, Entrez Gene IDs, RefSeq accessions, names, and Affymetrix probesets for the 325 genes are shown in Table S1. For genes with multiple Affymetrix probesets, a single probeset was selected to represent the gene. This was typically the probeset showing the highest expression measured in $\log 2$ signal intensity.

Sample information files were standardized for all 16 studies so that they could be combined conveniently into one large data structure. The individual studies were quantile-normalized using RMA in R and Bioconductor ("oligo" package), and expression levels were converted to the log base 2 scale (13).

Table 1. Sixteen cancer studies from GEO and ArrayExpress representing 8 cancer types.

\begin{tabular}{lcrc}
\hline Tissue & Accession & $\mathrm{N}^{1}$ & ${\text { Scale } \text { Factor }^{2}}^{\text {B }}$ \\
\hline Breast (BR1) & GSE25055 & 310 & 0.9760 \\
Breast (BR2) & E-TABM-157 & 51 & 1.0498 \\
Colon (CO1) & GSE39582 & 585 & 0.9660 \\
Colon (CO2) & GSE68468 & 366 & 0.8930 \\
Gastric (GA1) & GSE13911 & 69 & 1.0006 \\
Gastric (GA2) & GSE54129 & 132 & 0.9480 \\
Leukemia (LK1) & GSE13159 & 568 & 1.0129 \\
Leukemia (LK2) & GSE14471 & 110 & 1.1173 \\
Lung (LU1) & GSE19188 & 156 & 0.9731 \\
Lung (LU2) & GSE30219 & 307 & 0.9351 \\
Ovarian (OV1) & GSE26712 & 192 & 1.0915 \\
Ovarian (OV2) & GSE9891 & 285 & 0.9290 \\
Pancreatic (PA1) & GSE15471 & 78 & 0.9260 \\
Pancreatic (PA2) & GSE16515 & 52 & 0.9926 \\
Prostate (PR1) & GSE17951 & 154 & 0.7890 \\
Prostate (PR2) & GSE8218 & 148 & 0.8057 \\
\hline
\end{tabular}

${ }^{1}$ Number of samples from each study that were used in this analysis. ${ }^{2}$ Scale factor used for each study in batch correction.
The functional analysis of each gene (325) was generated from of the DAVID algorithm (the Database for Annotation, Visualization and Integration Discovery) (14). To describe significant canonical pathways, mutations, evidence of target drug development, biological functions, diseases, and interaction networks, QIAGEN's Ingenuity Pathway Analysis (IPA ${ }^{\circledR}$, QIAGEN, USA) was used. The core analysis was performed and direct and indirect relationships were considered to generate the networks. Canonical pathways were sorted by highest enrichment score and smallest Benjamini-Hochbergadjusted P-value.

\section{Cancer data sets}

Sixteen public data sets of microarray expression were selected for 8 cancer types: breast, colon, gastric, leukemia, lung, ovarian, pancreatic, and prostate cancers. The data sets were taken from NCBI's GEO and EBI's ArrayExpress resources $(15,16)$. The accession numbers and sample sizes used for each cancer type are shown in Table 1, adding up to 3,563 patients and cell lines. All of the microarrays used were Affymetrix HG-U133A or U133 Plus 2.0. These two platforms share 22,277 probesets in common, and only these common probesets were used in our analyses. The expression range of the 325 genes was examined in all 16 studies. In the 11 studies that included some normal samples (CO1, CO2, GA1, GA2, LK1, LU1, LU2, OV1, PA1, PA2, PR1), the classification of tumor and normal samples was tested. Seven of the studies included cancer subtype information (BR1, BR2, GA1, LK1, LU1, LU2, OV2) and these were used to test subtype classification.

For each classification test, feature selection was used to identify 20-gene subsets of the 325 genes that differed most between the two groups being compared and linear discriminant analysis (LDA) was used to classify the groups (17). Most of the studies are heavily unbalanced, having many more tumor samples than normal ones. In unbalanced cases like these, overall classification accuracy (number of correctly classified samples divided by all samples) is not a useful metric for evaluating model results, because a simple model classifying every sample as a tumor would be $95 \%$ accurate when $95 \%$ of the samples are tumors. Instead, a class accuracy average was used, where the accuracy of the tumor class was calculated first, then the accuracy of the normal class, and then these two values were averaged.

\section{Batch correction}

The 16 studies were batch corrected in order to compare gene expression levels across cancer types. Correction using ComBat (18) was attempted first, but covariates like disease subtype names were confounded with batches in several cases and ComBat would thus remove subtype variability. Instead, the 16 studies were batch corrected using an equal medians method, 
constraining the median of the 22,277 probesets to have the same value in each study (11). This preserves the variability of individual genes (like the 325-gene set) while putting the overall expression distributions of the 16 studies on a similar scale. The batch correction scale factor used for each study is included in Table 1.

\section{Randomized gene sets}

To test whether any random set of genes with the same size as the 325 genes would show similar expression profiles, 1,000 random sets of the 325 genes were sampled from the full list of 22,277 probesets. Total expression of each set was calculated as the sum of the average $\log _{2}$ intensities of the 325 genes. The gene expression of the 325 genes was also compared to a set of 325 randomly selected HK genes using the same calculations. The random $325 \mathrm{HK}$ genes were selected from only non-325 genes in Levanon's list of 3,804. Table $\mathrm{S} 1$ has two tabs, the first showing the 325 genes and the second the $102 \mathrm{HK}$ genes.

\section{Feature selection}

Subsets of the 325 genes that differed the most between tumor and normal conditions were identified by a feature selection method. Two values, TumorHigh and NormalHigh, were computed for each gene as follows: TumorHigh $=\left(\mathrm{T}_{\text {median }}-\mathrm{N}_{\text {median }}\right) /\left(\mathrm{T}_{\mathrm{SD}}+\mathrm{N}_{\mathrm{SD}}\right)$, NormalHigh $=\left(\mathrm{N}_{\text {median }}-\mathrm{T}_{\text {median }}\right) /\left(\mathrm{T}_{\mathrm{SD}}+\mathrm{N}_{\mathrm{SD}}\right)$. $\mathrm{T}_{\text {median }}$ and $\mathrm{N}_{\text {median }}$ are the median values of the tumor group and normal group, respectively, and $\mathrm{T}_{\mathrm{SD}}$ and $\mathrm{N}_{\mathrm{SD}}$ are their standard deviations. Genes with the top 10 TumorHigh scores were combined with genes having the top 10 NormalHigh scores, and these 20 genes were used as the features in the tumor/normal classification tests. The same feature selection method was used for the disease subtype tests, combining 10 genes higher in one subtype with 10 genes higher in the other subtype.

\section{Tumor vs normal tests}

LDA was performed to compare tumor and normal samples for the 11 studies that included normal samples (CO1, CO2, GA1, GA2, LK1, LU1, LU2, OV1, PA1, PA2, and PR1). Samples that were metastases or precancerous biopsies were excluded from the comparisons. Most of the 16 cancer data sets have heavily unbalanced groups being compared, such as 300 cancers compared to only 10 normal samples, which can bias differential expression results (19), and this is why differential expression was not used for our tests. LDA was used with equal prior probabilities, which avoided the bias of unbalanced groups.

\section{Disease subtype tests}

Seven of the studies (BR1, BR2, GA1, LK1, LU1, LU2, and OV2) contained cancer samples with different subtypes. The subtypes tested are listed in Table S2.
The same feature selection and LDA methods described above for the tumor vs normal tests were used for the subtype tests. In the R scripts for testing tumor/normal differences (tn_diffexp.R) and for testing subtype differences (e.g., ov2_subtypes.R), a maximum of twice as many samples was allowed in one group compared to another. For example, in ovarian cancer, if the input data had 100 serous samples and only 10 endometrioid samples, then 20 of the 100 serous samples were randomly selected to make the comparison 20 of one group vs 10 of the other group. This process was automated using $\mathrm{R}$ scripts so the rule was applied consistently to all comparisons made in the study.

\section{Contributions of HK genes}

The tumor vs normal and disease subtype tests were repeated using the HK subset of the 325 genes (102 genes) to examine whether these genes contributed to the separation of sample groups.

\section{Results}

\section{Expression of the $\mathbf{3 2 5}$ genes}

The expression levels of the 325 genes were plotted for each of the 16 studies. In all studies, the 325 genes were expressed across the full dynamic range of the Affymetrix platform, from $\log _{2}$ signal intensity values of about 4 to 12 . Using $\log _{2}<4$ as the background threshold, at least 324 of the 325 genes were expressed above background in every cancer type. Figure $\mathrm{S} 1$ shows boxplots of the 16 studies before (Figure S1A) and after (Figure S1B) batch correction. Figure S1C and D shows boxplots of the batch-corrected 325 and 102 HK genes.

The minimum, average, and maximum of the 1,000 expression testing results for the 325 genes are plotted for each cancer type in Figure S2. The 325 genes have higher total expression than all 1,000 random sets in every cancer type. The 325 genes also show more variability across cancer types than the random sets do. As another test, 325 other genes were selected at random from Levanon's 3,804 HK genes, where the random selection was constrained to prevent overlap with the 325 genes. The random HK genes were intermediate between the 325 genes and the random gene sets, both in their expression levels and in their variability across cancer types (Figure S2).

\section{Functional analysis of genes}

Functional analysis and grouping by biological function of the 325 genes was performed using DAVID bioinformatics resources (14). From the conversion of the Affymetrix probes to Entrez Format, the algorithm was able to return the function of 320 genes. The functional analysis of the genes is shown in Table S3. Furthermore, 67 gene clusters based on the greatest interactions and biological functions are provided. The clusters with the 
highest outstanding scores represent overlapping functions, for example, function in the cell cycle, cell division, mitosis, and DNA repair and replication (Table S4).

The analysis of genes using QIAGEN's Ingenuity Pathway Analysis (IPA ${ }^{\circledR}$, QIAGEN) shows the biological function of each gene. The software revealed diseases, expression profiles, molecular changes, and drugs under development that have the genes in question as a target for study (Table S5). In particular, the software has grouped the genes studied here into five major functional groups: cell survival and death $(P=5.00 \mathrm{E}-05)$, cell cycle $(P=6.92 E-05)$, DNA replication, recombination, and repair ( $P=6.92 \mathrm{E}-05)$, cell development $(P=7.76 \mathrm{E}-05)$, and cell growth and proliferation $(P=7.76 E-05)$, reaffirming the data found by the DAVID algorithm previously. The main diseases and disorders related to genes were five: cancer $(P=6.92 E-05)$, organismic injury and abnormalities ( $P=6.92 \mathrm{E}-05)$, gastrointestinal disease $(P=6.81 \mathrm{E}-05)$, reproductive system disease $(P=6.92 E-05)$, and endocrine system disorders $(P=3.68 E-05)$.

\section{Tumor vs normal tests}

The first test was to determine whether the gene panels were able to discriminate normal from tumor tissues. For that, 11 datasets were analyzed that contained both tumor and normal samples. Table 2 shows the tumor/normal classification results for the 325 and 102 genes. The LDA results show that the 325-gene panel correctly classified over $90 \%$ of tumor and normal samples in colon, gastric, leukemia, lung, ovarian, and prostate cancers datasets, and 4 of these datasets had higher than 95\% accuracy (CO2, GA2, OV1, PR1). Similar results were obtained using the two gastric (GA1, GA2) and pancreatic (PA1, PA2) cancer studies, but more variation is seen between the colon (CO1, CO2) and lung (LU1, LU2) cancer studies. When only the $102 \mathrm{HK}$ genes were used, the accuracy was still $90 \%$ or higher in the same datasets, and one pancreatic cancer set (PA2) that was $89 \%$ with 325 genes set moved up slightly to $91 \%$ with $102 \mathrm{HK}$ set, crossing the $90 \%$ threshold. These results showed the 325 and set of $102 \mathrm{HK}$ genes contributed to classifications of normal and tumor tissue in 8 of the 11 datasets analyzed, with accuracy above $90 \%$.

\section{Disease subtype tests}

Next, the 325 gene panel and the 102 HK subset were tested for tumor subtype classification ability. For this analysis, 14 comparisons were done using the 7 datasets that included tumor subtype information. Table 3 shows the cancer subtype classification results for the 325 and $102 \mathrm{HK}$ genes. The 325 genes had $90 \%$ or better accuracy in distinguishing one pair of leukemia subtypes (AML_NORM and AML_INVT), and three pairs of lung cancer subtypes (squamous vs adenocarcinoma in LU1, squamous vs large cell in LU1, and squamous vs adenocarcinoma in LU2). The $102 \mathrm{HK}$ genes showed reduced ability to distinguish tumor subtypes, reaching only $90.59 \%$ for LK1 (AML_NORM vs AML_INVT) and only $90.25 \%$ for LU1 (squamous vs large cell). In the breast cancer results, the comparisons between ER and TRIPNEG (ER positive and triple negative), ER/PR (ER or PR positive), and TRIPNEG in BR1 were very similar as would be expected ( $88 \%$ and $89 \%$, respectively), but the ER and TRIPNEG comparison in BR2 was very different $(59 \%)$. The BR1 samples came from 310 patients, whereas the BR2 samples are 51 cell lines, which is a likely explanation for the difference. In the lung cancer comparisons, squamous vs adenocarcinoma classification was high in both LU1 and LU2, but the subtypes that were only available in LU2 (squamous vs small cell, basaloid vs carcinoid) were poorly classified. Taken together, the results showed that the 325 panel can provide subtype classifications meeting the $90 \%$ threshold in one leukemia dataset and three lung cancer datasets

Table 2. Overall accuracy ("Overall"), tumor group accuracy ("TumorPct"), normal group accuracy ("NormPct"), and average of the tumor and normal class accuracies ("ClassAvg") for the 325-gene set and its 102 housekeeping (HK) subset.

\begin{tabular}{|c|c|c|c|c|c|c|c|c|}
\hline \multirow[t]{2}{*}{ Study/Gene Panel } & \multicolumn{2}{|c|}{ Overall } & \multicolumn{2}{|c|}{ TumorPct } & \multicolumn{2}{|c|}{ NormPct } & \multicolumn{2}{|c|}{ ClassAvg } \\
\hline & 325 & $\mathrm{HK}$ & 325 & $\mathrm{HK}$ & 325 & $\mathrm{HK}$ & 325 & $\mathrm{HK}$ \\
\hline $\mathrm{CO} 1$ & 84.23 & 80.64 & 84.65 & 81.33 & 73.68 & 63.16 & 79.17 & 72.24 \\
\hline $\mathrm{CO} 2$ & 98.4 & 98.4 & 98.97 & 98.97 & 96.36 & 96.36 & 97.67 & 97.67 \\
\hline GA1 & 94.2 & 95.65 & 92.11 & 94.74 & 96.77 & 96.77 & 94.44 & 95.76 \\
\hline GA2 & 100 & 100 & 100 & 100 & 100 & 100 & 100 & 100 \\
\hline LK1 & 91.9 & 92.78 & 90.89 & 91.9 & 98.65 & 98.65 & 94.77 & 95.28 \\
\hline LU1 & 94.23 & 94.23 & 94.51 & 93.41 & 93.85 & 95.38 & 94.18 & 94.4 \\
\hline LU2 & 80.46 & 73.94 & 81.57 & 75.77 & 57.14 & 35.71 & 69.36 & 55.74 \\
\hline OV1 & 100 & 100 & 100 & 100 & 100 & 100 & 100 & 100 \\
\hline PA1 & 87.18 & 83.33 & 92.31 & 79.49 & 82.05 & 87.18 & 87.18 & 83.33 \\
\hline PA2 & 86.54 & 90.38 & 83.33 & 88.89 & 93.75 & 93.75 & 88.54 & 91.32 \\
\hline PR1 & 98.36 & 95.9 & 98.18 & 96.36 & 100 & 91.67 & 99.09 & 94.02 \\
\hline
\end{tabular}

Red type indicates that the mean accuracy of 325 and 102 genes for LU2 dataset was less than $70 \%$. 
Table 3. Classification results for the 325-gene set and 102 housekeeping (HK) subset.

\begin{tabular}{|c|c|c|c|c|c|c|c|c|c|}
\hline \multirow[t]{2}{*}{ Study } & \multirow[t]{2}{*}{ Subtypes } & \multicolumn{2}{|c|}{ Overall } & \multicolumn{2}{|c|}{ Subtype1Pct } & \multicolumn{2}{|c|}{ Subtype2Pct } & \multicolumn{2}{|c|}{ ClassAvg } \\
\hline & & 325 & HK & 325 & $\mathrm{HK}$ & 325 & $\mathrm{HK}$ & 325 & HK \\
\hline BR1 & ER vs TRIPNEG & 89.76 & 84.94 & 84.09 & 75 & 91.8 & 88.52 & 87.95 & 81.76 \\
\hline BR1 & ER/PR vs TRIPNEG & 89.2 & 88.4 & 91.41 & 91.41 & 86.89 & 85.25 & 89.15 & 88.33 \\
\hline BR2 & ER vs TRIPNEG & 63.89 & 61.11 & 45.45 & 54.55 & 72 & 64 & 58.73 & 59.27 \\
\hline GA1 & MSI vs MSS & 84.21 & 68.42 & 78.95 & 63.16 & 89.47 & 73.68 & 84.21 & 68.42 \\
\hline LK1 & AML_NORM vs AML_INVT & 93.4 & 88.65 & 93.73 & 88.32 & 89.29 & 92.86 & 91.51 & 90.59 \\
\hline LK1 & AML_NORM vs AML_T1517 & 65.46 & 63.92 & 66.67 & 65.24 & 54.05 & 51.35 & 60.36 & 58.3 \\
\hline LK1 & AML_NORM vs AML_T821 & 63.94 & 64.71 & 65.53 & 66.95 & 50 & 45 & 57.76 & 55.98 \\
\hline LK1 & AML_NORM vs AML_TMLL & 59.64 & 59.9 & 60.68 & 61.82 & 50 & 42.11 & 55.34 & 51.96 \\
\hline LU1 & Squamous vs Adenocarcinoma & 98.61 & 88.89 & 96.3 & 81.48 & 100 & 93.33 & 98.15 & 87.41 \\
\hline LU1 & Squamous vs Large Cell & 100 & 91.3 & 100 & 96.3 & 100 & 84.21 & 100 & 90.25 \\
\hline LU2 & Basaloid vs Carcinoid & 92.06 & 88.89 & 89.74 & 92.31 & 95.83 & 83.33 & 92.79 & 87.82 \\
\hline LU2 & Squamous vs Adenocarcinoma & 52.74 & 61.64 & 52.46 & 63.93 & 52.94 & 60 & 52.7 & 61.97 \\
\hline LU2 & Squamous vs Small Cell & 69.51 & 70.73 & 73.77 & 75.41 & 57.14 & 57.14 & 65.46 & 66.28 \\
\hline OV2 & Serous vs Endometrioid & 92.96 & 90.85 & 94.7 & 91.67 & 70 & 80 & 82.35 & 85.83 \\
\hline
\end{tabular}

The "Subtype" columns list the two subtypes being compared. ER: estrogen receptor positive; ER/PR: estrogen and/or progesterone receptor positive; TRIPNEG: triple negative; MSI: microsatellite instability; MSS: microsatellite stability; AML_NORM: acute myeloid leukemia with normal karyotype; AML_INVT: AML with inv(16)/t(16;16); AML_T1517: AML with t(15;17); AML_T821: AML with t(8;21); AML_TMLL: AML with $t(11 q 23) / M L L$. Red type indicates that the mean accuracy of 325 and 102 genes for BR2, GA1, LK1, and LU2 datasets was less than $70 \%$.

whereas the $102 \mathrm{HK}$ followed the same trend but only met the threshold in half of these datasets.

\section{Discussion}

HK genes have been widely used for gene expression normalization due to their stable expression regardless of external and pathological conditions $(5,6)$. However, a recent evaluation of the expression of 32 genes classified as $\mathrm{HK}$ and applied to 12 different types of cancer has revealed that the GADPH gene (traditionally used as $\mathrm{HK}$ ) showed significant mRNA level alterations in more than half of the cases evaluated (18). These results revealed that HK expression may not follow the stability rule and still vary between tumors. In affirmation of this differential expression, the housekeeping genes of the ribosomal protein undergo variations for prostate cancer cells, as does the $\beta$-actin gene for treated colorectal cells (20).

In this context, in the healthy tissues where the 325 genes were originally identified (8), these genes were upregulated in the disorganized proliferating state and downregulated in the organized and growth-arrested state. An unexpected result is that of these genes, 102 were HK genes, showing biomarker potential for HK. A second unexpected result was that these differences extended to at least seven other cancer types (colon, gastric, leukemia, lung, ovarian, pancreatic, and prostate). This was consistent with our results showing their expression differences between tumors and normal tissues (see Table 2).

Before this study, it was not recognized that about one third of the 325 genes qualify as HK genes based on their relatively stable expression across tissues. Yet, HK genes were sufficient to separate tumors from normal tissue in seven of the cancer types (colon, gastric, leukemia, lung, ovarian, pancreatic, and prostate), without contributions from non-HK genes. The functions of the HK subset of the 325 genes include mRNA splicing, mRNA export from the nucleus, regulation of response to heat, recruitment of factors to DNA lesions, protein import into the nucleus, and mitochondrial genome maintenance (Tables S3 and S4).

The expression results showed that the 325 genes were expressed at a broad range of levels in 8 different cancer types (breast, colon, gastric, leukemia, lung, ovarian, pancreatic, and prostate) and at higher levels than randomly selected gene lists, despite being originally identified in a breast-specific developmental process. The genes were able to accurately classify tumor and normal samples from colon, gastric, leukemia, lung, ovarian, and prostate cancers, and some subtypes of lung cancer and leukemia. The HK subset was able to classify tumor and normal samples in all of the same cancer types as the 325 set as well as pancreatic cancer, while showing reduced accuracy in subtype classification. These lines of evidence support the potential utility of both the 325 genes and the 102 HK genes as biomarkers in multiple cancer types. Recently, our group showed that the differential expression of 325 genes in breast biopsies in neoadjuvant chemotherapy were able to stratify, surprisingly, the cases with RD at a rate of 83 and $91 \%$ in two independent cohorts (519 and 304 cases), comprising different breast tumor subtypes (ER +HER2-, triple negative, HER2+, and ER-HER-PR+). Furthermore, for the triple negative group, a tumor subtype 
with a worse prognosis and high rates of recurrence, the 325 genes in two different cohorts were able to identify $85.4 \%$ (88/ 103 ) and $86.2 \%(56 / 65)$ of the cases with RD (9).

This study is original and expands with multiple results from in silico analysis. Further work is needed to validate these putative tumor-specific markers for independent experimental and clinical validations, including different tumor subtypes and their molecular variations. The research opens avenues for investigation of other HK genes outside the set of 325 genes that could also classify tumors and normal samples. In addition to tumor/normal classification, further work will focus on specific predictions of interest (diagnosis, prognosis, patient stratification) in order to confirm that the 325 and 102 genes can provide clinical benefits.

\section{References}

1. Bissell MJ, Radisky DC, Rizki A, Weaver VM, Petersen OW. The organizing principle: microenvironmental influences in the normal and malignant breast. Differentiation 2002; 70 : 537-546, doi: 10.1046/j.1432-0436.2002.700907.x.

2. Petersen OW, Rønnov-Jessen L, Weaver VM, Bissell MJ. Differentiation and cancer in the mammary gland: shedding light on an old dichotomy. Adv Cancer Res 1998; 75: 135-161, doi: 10.1016/S0065-230X(08)60741-1.

3. Polyak K, Kalluri R. The role of the microenvironment in mammary gland development and cancer. Cold Spring Harb Perspect Biol 2010; 2: a003244, doi: 10.1101/cshperspect. a003244.

4. Seth P, Porter D, Lahti-Domenici J, Geng Y, Richardson A, Polyak K. Cellular and molecular targets of estrogen in normal human breast tissue. Cancer Res 2002; 62: 4540-4544.

5. Eisenberg E, Levanon EY. Human housekeeping genes are compact. Trends Genet 2003; 19: 362-365, doi: 10.1016/ S0168-9525(03)00140-9.

6. Eisenberg E, Levanon EY. Human housekeeping genes, revisited. Trends Genet 2013, 29: 569-574, doi: 10.1016/ j.tig.2013.05.010.

7. Fournier MV, Martin KJ, Kenny PA, Xhaja K, Bosch I, Yaswen $P$, et al. Gene expression signature in organized and growth-arrested mammary acini predicts good outcome in breast cancer. Cancer Res 2006; 66: 7095-7102, doi: 10.1158/0008-5472.CAN-06-0515.

8. Martin KJ, Patrick DR, Bissell MJ, Fournier MV. Prognostic breast cancer signature identified from 3D culture model accurately predicts clinical outcome across independent datasets. PLoS One 2008; 3: e2994, doi: 10.1371/journal. pone.0002994.

9. Fournier MV, Goodwin EC, Chen J, Obenauer JC, Tannenbaum SH, Brufsky AM. A predictor of pathological complete response to neoadjuvant chemotherapy stratifies triple negative breast cancer patients with high risk of recurrence. Sci Rep 2019; 9:14863, doi: 10.1038/s41598-019-51335-1.

10. Delmonico L, Attiya S, Chen JW, Obenauer JC, Goodwin EC, Fournier MV. Expression concordance of 325 novel RNA biomarkers between data generated by NanoString nCounter and Affymetrix GeneChip. Dis Markers 2019; 940347, doi: 10.1155/2019/1940347.

\section{Supplementary Material}

Click to view [pdf].

\section{Acknowledgments}

L. Delmonico received an international sandwichdoctorate scholarship (Process No. 88881.123875/201601) financed by Coordenação de Aperfeiçoamento de Pessoal de Nível Superior (CAPES). J.C. Obenauer is employed by Rancho BioSciences and T.P. Stockfisch performs contract work for Rancho BioSciences. This research was supported by Bioarray Genetics Inc.

11. Obenauer JC, Stockfisch TP, Fournier MV. Overcorrection of batch effects by ComBat can be avoided by using an equal medians method. Proceed Am Assoc Cancer Res Annual Meet 2019; 79, doi: 10.1158/1538-7445.AM2019-1659.

12. Neve RM, Chin K, Fridlyand J, Yeh J, Baehner FL, Fevr T, et al. A collection of breast cancer cell lines for the study of functionally distinct cancer subtypes. Cancer Cell 2006; 10 , 515-527, doi: 10.1016/j.ccr.2006.10.008.

13. Bolstad BM, Irizarry RA, Astrand M, Speed TP. A comparison of normalization methods for high density oligonucleotide array data based on variance and bias. Bioinformatics 2003; 19: 185-193, doi: 10.1093/bioinformatics/19.2.185.

14. Huang DW, Sherman BT, Tan Q, Kir J, Liu D, Bryant D, et al. DAVID Bioinformatics Resources: expanded annotation database and novel algorithms to better extract biology from large gene lists. Nucleic Acids Res 2007; 35: W169W175, doi: 10.1093/nar/gkm415.

15. Barrett T, Wilhite SE, Ledoux P, Evangelista C, Kim IF, Tomashevsky $\mathrm{M}$, et al. NCBI GEO: archive for functional genomics data sets--update. Nucleic Acids Res 2013; 41: D991-D995, doi: 10.1093/nar/gks1193.

16. Kolesnikov N, Hastings E, Keays M, Melnichuk O, Tang YA, Williams $\mathrm{E}$, et al. ArrayExpress update-simplifying data submissions. Nucleic Acids Res 2015; 43: D1113-D1116, doi: $10.1093 /$ nar/gku1057.

17. Huang D, Quan Y, He M, Zhou B. Comparison of linear discriminant analysis methods for the classification of cancer based on gene expression data. J Exp Clin Cancer Res 2009; 28: 149, doi: 10.1186/1756-9966-28-149.

18. Krasnov GS, Kudryavtseva AV, Snezhkina AV, Lakunina VA, Beniaminov AD, Melnikova NV, et al. Pan-Cancer analysis of TCGA data revealed promising reference genes for qPCR normalization. Front Genet 2019; 10: 97, doi: 10.3389/ fgene.2019.00097.

19. Yang K, Li J, Gao H. The impact of sample imbalance on identifying differentially expressed genes. BMC Bioinformatics 2006; 4: S8, doi: 10.1186/1471-2105-7-S4-S8.

20. Khimani AH, Mhashilkar AM, Mikulskis A, O'Malley M, Liao $J$, Golenko EE, et al. Housekeeping genes in cancer: normalization of array data. Biotechniques 2005; 38: 739745, doi: 10.2144/05385ST04. 